\title{
CULTURA MATERIAL Y FUENTES ESCRITAS: LOS CHICHAS DE LOS ANDES DEL SUR.
}

\author{
Maria Beierlein de Gutiérrez
}

Instituto Latinoamericano, Berlín, Alemania.Simplonstrasse 8, 10245 Berlín, Alemania.MariaBeierlein@gmx.de

Presentado: 18/06/2007- Aceptado: 28/04/2008

\begin{abstract}
Resumen
Durante los últimos años el desarrollo de la investigación arqueológica sobre el tipo cerámico Yavi-Chica y el estudio de las fuentes documentales del siglo XVI sobre una entidad cultura denominada Chichas, fue construyendo un escenario conflictivo al momento de realizar su comparación. En el presente artículo se abordará dicha problemática, con el objetivo de proponer la existencia de la cerámica Yavi-Chicha asociada a un determinado territorio con diversas subvariantes estilísticas y la presencia de una entidad cultural Chichas. Tanto la información arqueológica y los datos presentes en las fuentes documentales, nos proporcionaran importantes indicadores sobre la cultura material y las relaciones etnohistóricas de los Chichas, ademas de intentar aclarar el desfase entre el uso de los conceptos coloniales referente a "etnias" $y$ "naciones" $y$ de los grupos de identidad correspondientes a las poblaciones que habitaban este territorio. En este sentido es posible afirmar que las relaciones heterogéneas y activas entre ellos corresponden a diferentes contextos sociopolíticos a lo largo del tiempo y representan un amplio panorama de interrelaciones humanas.
\end{abstract}

Palabras ClavesChichas, Andes Centro Sur, Etnohistoria, Arqueología

\begin{abstract}
In recent years, archaeological investigation of the Yavi-Chicha ceramic type and the study of the documentary sources of the XVI century about a cultural entity called Chichas has created a conflictive panorama when it came to comparing both sources. The present article presents an overview over the problematic situation and suggests that the existence of the Yavi-Chicha ceramic is linked to a specific territory with a range of stylistic varieties and the presence of the cultural entity called Chichas. Archaeological findings as well as documentary sources allow important insights into the material culture and the ethnohistoric relationships of the Chichas. They also demonstrate the differences as to the use of the colonial concepts of "etnias" and "naciones" and the identity groups which correspond to the persons which were living in this territory. Using both sources of data it will be possible to affirm that the heterogeneous and active relationships between them correspond to different sociopolitical contexts evolving during the existence of this territory, representing a wide panorama of human interrelationships.
\end{abstract}

Keywords Chichas, South Central Andes, Ethnohistory, Archaeology 


\section{Introducción}

Durante los últimos años las referencias arqueológicas e históricas a los Chichas como una entidad cultural fueron aumentando, pero esto llevó a una considerable confusión sobre el carácter de su cultura material reflejado en los datos arqueológicos y la relación con los datos etnohistóricos.

Esto se debe en parte a que las referencias arqueológicas postulan la presencia de la cerámica Yavi-Chicha en diferentes regiones y/o áreas muy limitadas y en un período de tiempo dado y por otra parte los datos etnohistóricos contextualizan la presencia de los Chichas en una región "nuclear"en el Sur de Bolivia y sus relaciones con el Noroeste Argentino ${ }^{1}$.

En este trabajo intento proponer una síntesis entre los dos juegos de datos, proponiendo una visión que une los datos arqueológicos y etnohistóricos, para demostrar las superposiciones y desfases y tratar de encontrar explicaciones para esta problemática. Sin embargo, aunque no es posible solucionar completamente estas contradicciones, sí podemos ampliar nuestra visión de "Los Chichas" tanto en la arqueología como en la etnohistoria, mediante una revisión detallada de los datos existentes. Sostengo la idea de un territorio de influencia Chicha caracterizado por relaciones internas heterogéneas y activas, correspondientes a diferentes contextos sociopolíticos expresados mediante grupos de identidad en un amplio panorama de relaciones humanas.

Ya que la arqueología nos brinda una profundidad temporal mayor, propongo la separación analítica entre un momento preincaico - investigado arqueológicamente - y el período de influencia inca, representado por la arqueología y los documentos coloniales.

\section{Identidad cultural, etnicidad, cultura material y grupos de identidad - hacia una definición de "lo Chicha"}

El trabajo con datos acerca de un supuesto "grupo étnico", sea este definido arqueológicamente o históricamente, lleva a la pregunta acerca de la continuidad de conceptos de etnicidad e identidad cultural en los diferentes juegos de datos (p.e. Bernbeck 1997, Knapp 1992, Wilson 1993). Aunque se puede decir que la etnohistoria ha trabajado estos problemas, definiendo términos como "cultura", "etnicidad" e "identidad cultural" según sus específicos contextos históricos, la arqueología sufre todavía de las contradicciones y confusiones entre estos conceptos.

Varios autores han ofrecido definiciones y criticas al respecto, especialmente acerca de la supuesta continuidad entre grupos étnicos de las fuentes documentales coloniales y la cultura material investigada por los arqueólogos. Los términos de "etnicidad" e "identidad cultural" se usan como sinónimos (Graves-Brown et al. 1996, Jones 1997, Shennan 1989), sin embargo, se trata de dos conceptos distintos. Se discute la existencia del concepto de "etnicidad" en el tiempo prehispánico (o prehistórico en el caso de Europa) por lo que se ha optado por usar el concepto de "identidad cultural". Las expresiones de etnicidad (Barth 1969, Hodder 1982) se relacionan con componentes de la identidad cultural, expresada por elementos específicos de la cultura material. Este uso situacional llevó a una imagen transeúnte e inestable de la etnicidad (ver también Wallman 1977 citado en Jones \& Graves-Brown 1996, pág. 6). 
La identidad - y con ella la identidad cultural - es un constructo social, siempre relacionada con el mundo exterior que nos rodea, manifestándose arqueológicamente en la cultura material. La identidad no es estable, sino se entiende mas bien como fragmentada, formada por diferentes grupos de identidad pertenecientes a un solo actor social. Estos grupos de identidad pueden tener diferentes profundidades temporales (Cornell 2004). De esta manera es posible entender las diferentes expresiones materiales como relacionadas con diferentes grupos de identidad, los cuales tienen duraciones temporales distintas. Mientras que algunos de ellos pueden ser de muy corta duración, usadas durante un momento social específico, otras pueden asociarse a ideas como etnicidad o sistemas de parentesco (ibd., pág. 78).

Si seguimos la propuesta de los grupos de identidad, las diferentes expresiones materiales serían su contraparte arqueológica y pueden ser analizadas como tales. En este sentido la cerámica Yavi-Chicha constituiría la expresión material de un grupo de identidad prehispánico regional y probablemente con una profundidad temporal mayor, sus diferentes variantes temporales y locales podrían ser elementos representativos de diferentes grupos de identidad al interior del grupo principal o estar relacionados con diferentes fines sociopolíticos o ideológicos.

\section{Los Chichas en las fuentes documentales del siglo XVI}

\section{Naciones y provincias}

La región al Sur del lago Titicaca está representada sobre todo por probanzas de méritos, documentos de encomienda, memoriales y pleitos, estos documentos se remiten a informaciones de la población local y los escribanos locales involucrados. El documento más detallado es el Memorial de Charcas (1969[1582]), el cual fue redactado con el fin primordial de protestar contra la tasa de Toledo. Sin embargo, la autorepresentación de los curacas indígenas forma parte integral de una coyuntura sociopolítica y sigue ciertos intereses (Julien 1999, Pease 1995). Según los datos ofrecidos por los curacas de la federación Charcas (Espinoza Soriano 1969, pág. 18) siete naciones formaron esta federación: los Chichas, Chuis, Charcas y Caracaras (o Qaraqara), Soras, Quillacas y Carangas junto con otros grupos como los Yamparas (Julien 1995) y sus respectivos subgrupos.

Los documentos etnohistóricos presentan al grupo Chichas como una nación supuestamente homogénea y los curacas usan este término para su autorepresentación en el Memorial de Charcas. Sin embargo, este hecho es cuestionable a la luz de otras fuentes donde se mencionan diferentes grupos existentes al interior del territorio Chicha. Por esto es probable que se trate de un territorio donde los Chichas cohabitaban con otros grupos, además de tener una intensiva relación con grupos vecinos dentro de la federación Charcas. Por lo tanto la palabra nación podría haber sido usada de manera estratégica, intentando una visión más "europea" de los respectivos grupos. El término "provincia" también aparece en las fuentes tempranas, teniendo más bien un sentido étnico y político en vez de geográfico (Julien 1993, Pease 1995), aplicándose a grupos étnicos muchas veces conquistados por los Incas.

Sin embargo, las palabras nación y provincia usadas en diferentes textos coloniales parecen indicar que la provincia Charcas y con ella los Chichas poseían una estructura política definida, aunque se discute si este patrón corresponde a la influencia inca en la zona o a 
patrones organizativos pre-incaicos. Además se diferenciaban probablemente en parcialidades de hanan y hurin como se menciona en la Tasa de Toledo (1975 [1573], pág. 27), con una parcialidad en Talina y la otra en Calcha, un dato también propuesto por Saignes (1986), y también por la lista de encomienda de Hernando Pizarro (Varón Gabai 1997).

\section{Ubicación geográfica - perspectiva macroregional o enfoque regional?}

Espinoza Soriano (1969, pág. 6) sospechaba la ubicación de los Chichas al Sur de Potosí, entre los Lípez, Carangas y Chiriguanos. Para Presta (1995), el área nuclear de los Chichas se encontraría alrededor del río San Juan de Oro y del río Camblaya al Norte de Tarija. Los datos acerca de los márgenes orientales y australes del territorio Chicha conducen al tema de las supuestas fronteras "étnicas", que sufre de ambivalencias en cuanto a sus definiciones terminológicas y territoriales. Especialmente las fronteras orientales de los Andes son tema de debate, incluyendo la frontera Chicha con la vertiente oriental de los Andes. Las fluidas fronteras con los Chiriguanos y las nociones culturales implícitas en la representación indígena y española de la frontera oriental como un límite "cultural" entre tierras altas y bajas (Garay de Fumagalli 2003, Renard Casevitz y Saignes 1988) complican el panorama geográfico visible en las fuentes escritas. Existen fuentes que ubican los Chichas en la frontera de los Chiriguanos en el siglo XVI (Varón Gabai 1997), donde según ellos mismos habían sido mandados por los Incas para resguardar las fronteras orientales del estado Inca (Espinoza 1969, Zanolli 2003), y las fortalezas de la vertiente oriental de los Andes se asocian muchas veces con los Chichas y otras etnias relacionadas con ellos. La autorepresentación de los Chichas como supuestos "defensores" de las tierras altas se encuentra en concordancia con la idea colonial sobre la dicotomía entre tierras altas y bajas, como ejemplos de lo "civilizado" y lo "bárbaro". Existe una intensiva interacción de diversos grupos dentro del territorio Chichas y su frontera oriental, entre ellos los Chichas, Churumatas, Chuis, Moyo-moyos y Ocloyas, que demuestran el activo relacionamiento con grupos de territorios más orientales, la permeabilidad de la zona fronteriza y la intencionalidad de la presentación hermética de la vertiente oriental ${ }^{2}$

El límite Sur del territorio Chicha fue propuesto por Zanolli (1995) en la región de Sococha y el territorio Omaguaca, al norte de Argentina y sur de Bolivia, especialmente el valle de Tarija. El autor propone una presencia esporádica de los Chichas en la Puna Argentina y la Quebrada de Humahuaca y desarrolla la idea de regiones multiétnicas (Albeck 2003).

Los datos etnohistóricos acerca del límite occidental del territorio Chicha son extremadamente limitados y se remiten a unas menciones en la "Relación..." de Vivar ([1558] 1979) y datos ofrecidos por Zanolli (2003). De esto podemos rescatar la idea que los caciques Chichas ejercían un cierto grado de dominación sobre grupos chilenos, especialmente en el área de Atacama.

Según los datos etnohistóricos podemos proponer entonces un área de población que se extiende del Sur de Potosí, posiblemente desde Calcha hacia el Sur, Lípez al Este, los Chiriguanos al Oeste ${ }^{3} y$ la Puna Argentina al Sur, teniendo su posible centro alrededor de la cuenca del río San Juan del Oro y la cuenca de Yavi.

"... hemos sido soldados desde el tiempo de los ingas" 
En cuanto a la organización sociopolítica existe la visión que nos ofrecen los curacas de la federación Charcas mediante el "Memorial de Charcas":

"En nuestra prouincia de Los Charcas, antes de los ingas y despues de ellos solia hauer señores naturales mayores de a diez mil vasallos y otros de a ocho mil indios y otros de a seis mil indios y vasallos otros dichos señores y caualleros eran superiores de los demás caciques y señores que hauía en cada nación. [...] Y ansi cada uno de estos señores solían tener ocho segundas personas y diez también de a mil indios y cuatro principales de cada ayllu de a quinientos y de cient indios y cuatro mandones en cada ayllu cada uno en su nación de hanansaya y Hurinsaya." (Memorial... 1969 [1582], pág. 18).

Según las fuentes los Chichas se diferenciaban en parcialidades de hanan y hurin (Cook 1975 [1573]), con una parcialidad en Talina y la otra en Calcha (Saignes 1985, Espinoza 1969). La lista de la encomienda de Hernando Pizarro da una impresión de la diversidad de mitmaqkuna en el territorio Chicha ${ }^{4}$, donde podemos observar a mitmaqkuna Carangas y Quillacas de la federación de Charcas, Canches y Collas del área circuntiticaca, mitmaqkuna del Cuzco, del Cuntisuyu y de Ollantaytambo (com. pers. Kerstin Nowack 2006). Además existe un intenso debate sobre grupos que posiblemente cohabitaban con los Chichas o mantenían relaciones con ellos (Barragán 1994, Pärssinen 2003). Aunque estos datos se refieren sobre todo a la época incaica y colonial, existen fuentes que hablan del proceso de conquista inca. Zanolli (2003) observa que los grupos del Altiplano Sur se opusieron a la conquista incaica mediante alianzas de guerra. Los Incas respondían con la conquista militar que terminó en la anexión de la Provincia de Charcas y su integración al régimen incaico ${ }^{5}$. Según el "Memorial..." ([1582]1969) los Incas dieron el titulo de Orejones a los Chichas (ver también Zanolli 2003) y se ha propuesto el refuerzo de las relaciones entre Incas y Chichas mediante el intercambio de mujeres y matrimonios políticos entre ambos (Pärssinen 2003, Williams 2004).

En el Memorial de Charcas los Chichas hacen hincapié en presentarse como parte de un grupo de élite del ejército inca:

„Y es ansi que estas dichas cuatro naciones [Charcas, Caracaras, Chuyes, Chichas] [...] hemos sido soldados desde el tiempo de los ingas referido arriua, reseruados de pechos y alcaualas y de todas las demas tasas y seruicios [...] má[s] que no éramos gente de bailadores ni truhanes que estos tales tenían de costumbre de cantar canciones delante de los dichos ingas por las victorias cuando nosotros las dichas cuatro naciones vencíamos y teníamos victoria contra los Chachapoyas, cayambis, cañares, quitos y quillaycincas [...]. (Memorial..., 1969[1582], pág. 24).

Según el "Memorial...", los grupos mencionados se empleaban en el resguardo de la frontera incaica "en las fronteras y guarniciones en las fortalezas contra los chiriguanaes." (ibd., pág. 24). La autorepresentación como grupo belicoso se refleja también en la continuidad de ser usados como guerreros bajo los españoles cuando se formó una excursión contra los Chiriguanos (ibd., pág. 22ff).

Hay que destacar que la situación en la frontera oriental meridional del estado Inca parece corresponder a patrones de poblamiento específicos. Aparte de los Chichas, los Charcas, Qaraqaras y Chuis sirvieron en las fortalezas incaicas, juntos con los Carangas 
(Saignes 1985), los Churumatas6, los Moyo-moyos (Julien 1995, Presta 1995, Saignes 1985) y Yamparas (Julien 1995). Aunque se supone que los Charcas, Carangas y Qaraqaras dejaron las fortalezas poco después de la llegada de los españoles (Presta 1995), la situación de los Chichas y los demás grupos parece ser distinta ya que sus territorios se encuentran en proximidad a la frontera con los Chiriguanos y es posible que ellos siguieran en resguardo de las fronteras por su propio interés. Esta idea encuentra resonancia en el hecho de que el servicio en las fronteras chiriguanas se prolongó en tiempo colonial. En el "Memorial de Charcas" se observa acerca de la mit’ a colonial que:

"... [se] repartió y señaló tan solamente indios para el beneficio y labor de las minas e ingenios en la Uilla de Potosí a la nación de Los Charcas, Caracaras, Soras, Quillacas y Carangas, reservando como reservó sola la nación de Los Chichas y a la nación de Los Chuis y Amparaes [...] y ellos siruen tan solamente en la ciudad de la Plata y en la Uilla de Tarija y en la Uilla de Tomina que son las fronteras con los chiriguanaes." (Memorial..., pág. 22).

Según esta fuente el resguardo de la frontera chiriguana era la única y exclusiva mit’a incaica que tenían que proporcionar los Chichas. Sin embargo, existen otros datos donde son mencionados en relación con la explotación de minas de oro y plata (Cieza de León [1553]1984, Lorandi 1984, Schramm 1990) y se ha propuesto la posibilidad de haber ocupado posiciones de supervisión en Ocloya (Lorandi 1984, Zanolli 2003).

Las fuentes presentan un panorama muy heterogéneo de los Chichas. Podemos detectar un territorio nuclear al Sur de Potosí, limitando al Este con los Chiriguanos, al Oeste con Lípez y al Sur con la Puna Argentina. Aparte de esta región nuclear se deduce la interacción con otros grupos, tanto altiplánicos como de la vertiente oriental andina, indicando diferentes formas de interacción y relacionamiento. Las fuentes presentan cada relación inter- e intraregional como correspondiente a una situación sociopolítica específica, en muchos casos ligado a la influencia del estado inca sobre los diferentes grupos regionales. Estas diferentes situaciones sociopolíticas podrían haber formado la base para el desarrollo y uso de diferentes grupos de identidad, definidos geográficamente y/o en el transcurso de la interacción entre diferentes entidades sociales como las supuestas "etnias" de las fuentes etnohistóricas.

\section{Los Chichas en el material arqueológico: identidades heterogéneas}

En los últimos años los territorios adscritos a los grupos de la federación de Charcas se contrastaron con los datos arqueológicos, indicando posibles límites sociopolíticos compartidos entre la arqueología y la etnohistoria (p.e. Catacora et al. 2006, Michel 2000, Platt et al. 2006).

Tradicionalmente se buscaron sitios mencionados en las fuentes o se adscribieron territorios a grupos etnohistóricos mediante el análisis de los restos materiales como indicadores de "culturas" estilísticamente definibles. Ya hemos discutido la validez que relacione un grupo supuestamente "étnico" a una posible representación en la cultura material, formando "límites culturales" representados en supuestos "límites estilísticos". En vez de entender estos grupos como "etnias" o "culturas" estáticas, es preferible permitir interpretaciones más abiertas, proponiendo la actuación de la cultura material dentro de una sociedad como "referente a" y "reflejo de" contextos sociopolíticos e ideológicos 


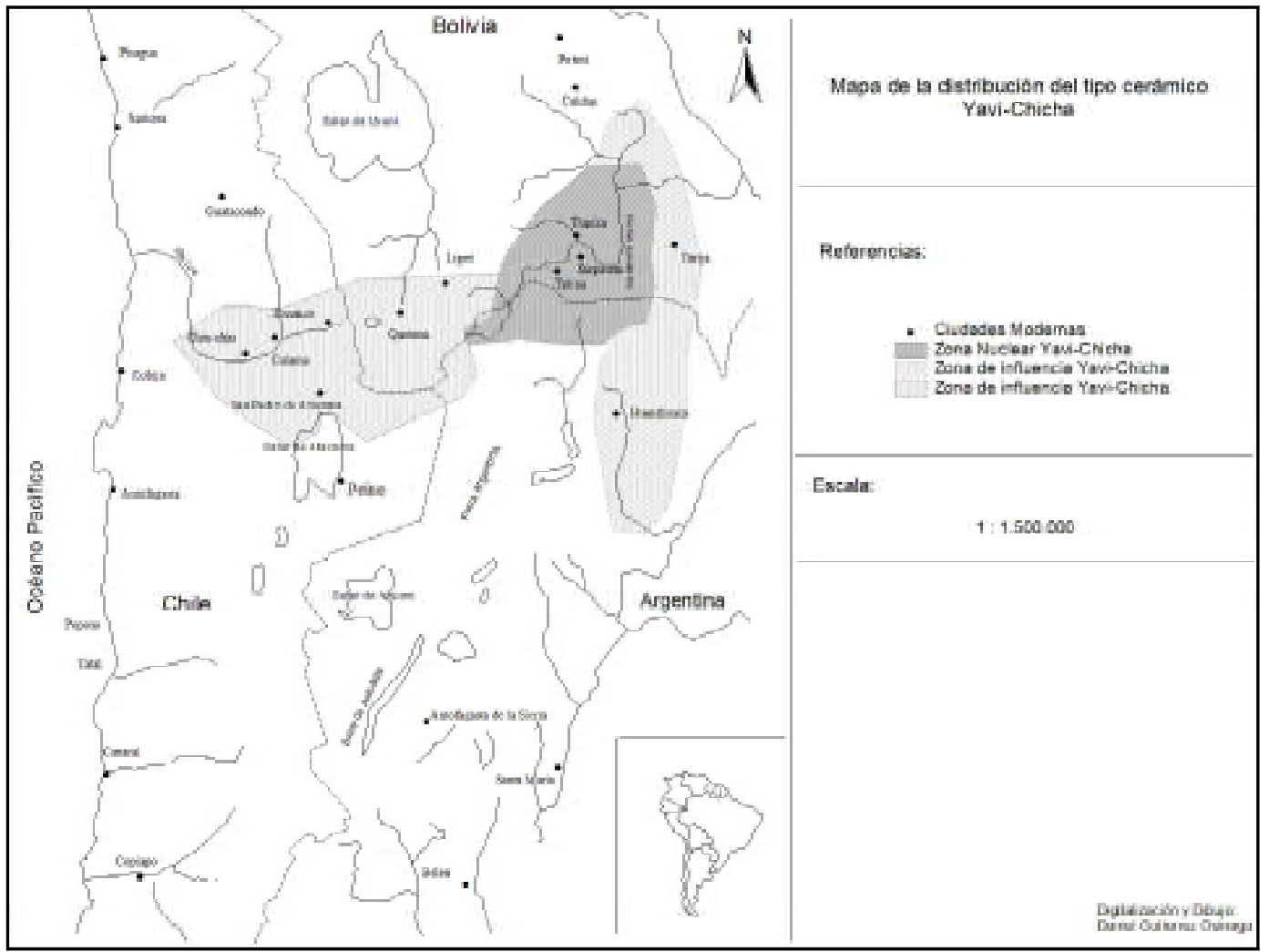

Figura 1. Mapa de la distribución del tipo cerámico Yavi-Chicha

específicos y múltiples (Chilton 1999, Shanks y Tilley 1992), llegando a una multicontextualidad de los bienes (Fahlander y Oestigaard 2004, Tilley 1989). De esta manera podemos entender las diferentes variedades de la cultura material Chicha como expresión de diferentes grupos de identidad, correspondientes a diferentes situaciones sociopolíticas.

El término Chicha se refiere aquí a un estilo definido en el material cerámico encontrado mayormente en la región del Altiplano Meridional de Lípez, el río San Juan del Oro, el Altiplano de Sama y la Puna de Jujuy, llamado Yavi (Krapovickas 1965, 1975, Krapovickas et al. 1989) o Chichas (Ibarra Grasso y Querejazu 1986) y, últimamente, Yavi-Chicha (Angelo 1999, 2006, Beierlein 2004, 2006, Uribe 1997) ${ }^{7}$.

Las investigaciones arqueológicas demuestran una concentración en tres focos de investigación: trabajos en el territorio nuclear Chicha que tenían como objetivo investigar la ocupación prehispánica en general o de períodos específicos, los trabajos en regiones aledañas al territorio Chicha, donde material cerámico de filiación Yavi-Chicha es reportado como parte de una amplia gama de estilos y, tercero, las investigaciones en sitios específicos asociados con los Chichas en las fuentes escritas, muchas veces relacionado con el período inca. 


\section{Cerámica Yavi-Chicha antes de los Incas}

El estilo Yavi-Chicha, muchas veces propuesto como contraparte material del grupo etnohistórico Chichas (Krapovickas 1965, 1975, 1984, Krapovickas et al. 1989, Ibarra Grasso y Querejazu 1986), se caracteriza por una cerámica de colores claros (naranja, rojo, morado, ante) con inclusiones blancas, cubierto de un engobe claro (anaranjado, rojo, morado, ante o de color de la pasta), decorado con complejos motivos geométricos en forma de espirales, volutas, triángulos, líneas paralelas etc. (Fig. 2a). Este estilo fue descrito como Yavi para la Puna Argentina, proponiendo el valle del San Juan del Oro y el grupo de los Chichas prehispánicos como punto de origen y como Cultura Chicha en Bolivia, localizado entre Tarija, Camargo, Tupiza y el Sur de Potosí. Krapovickas define diferentes variantes, las cuales destacan por sus diferentes atributos decorativos o tecnológicos, otros autores (p.e. Raffino et al. 1993) han ampliado la gama de variedades de la cerámica Yavi-Chicha.

Cerámica Yavi-Chicha ha sido reportada para la región de Lípez (Nielsen 2003, 2004), el valle del San Juan del Oro (Angelo 1999), el Altiplano de Sama (Beierlein 2004, 2006), dejando rastros también en el valle de Tarija (Rendón 2004) ${ }^{8}$. Sin embargo, la cerámica del Valle de Tarija pertenece a cánones decorativos y tecnológicos muy diferentes (Arellano 1984, Rendón 2004). En las regiones al Norte (Lecoq 1999, Rivera 2005) el estilo Yavi-Chicha no está presente en el Período de Desarrollos Regionales Tardíos.

En la región nuclear Chicha la influencia Tiwanaku no es visible en el registro arqueológico, sugiriendo la posibilidad que la cerámica Yavi-Chicha sea el directo sucesor de los estilos formativos en esta región, desarrollándose en el Periodo de Desarrollos Regionales Tempranos. Existen contactos con la región atacameña (Uribe 2002) y los grupos de la Quebrada de Humahuaca (Nielsen 1996), donde cerámica de características Yavi-Chicha aparece desde el Período Medio ${ }^{9}$, lo que nos da un terminus ante quem para el desarrollo en la zona nuclear.

Podemos proponer entonces una distribución preincaica de cerámica Yavi-Chicha entre la Puna Argentina Septentrional, el valle del Río San Juan del Oro, el Altiplano de Sama y la parte Sur del Altiplano de Lípez. Esta macroregión fue propuesta también por Nielsen (2003), quien la entiende como un "sistema regional" de varios pisos altitudinales controlado por un grupo asociado con cerámica Yavi-Chicha. Existe además cerámica Yavi-Chicha en San Pedro de Atacama y el valle del río Loa (Cantarutti y Mera s.a., Tarragó 1977, 1989 citado en Ávila 2005, Uribe 1997, 2002) y es probable que la introducción de ésta se relacione con el intercambio continuo de bienes e ideas entre las zonas del Norte de Chile, la Puna y los valles y quebradas orientales al Este (Angelo 2006, Angelo y Capriles 2004, Nielsen 2004, Núñez y Dillehay 1995 [1979]). Los datos arqueológicos sugieren que este intercambio tenía una profundidad temporal prolongada y forma la base de interrelacionamiento entre los diferentes grupos de este amplio territorio.

Un caso especial son las regiones orientales. La falta de investigaciones y dataciones confiables impide definir si existían contactos con el área nuclear Chicha antes de los Incas, pero se observa que la mención de los grupos supuestamente orientales juntos a los Chichas en la mit’a inca se homologa con el registro arqueológico. La existencia de cerámica YaviChicha fue interpretada como indicador de relaciones con el área nuclear y cerámica supuestamente local como testigo de la presencia de grupos de la vertiente oriental ${ }^{10}$. 


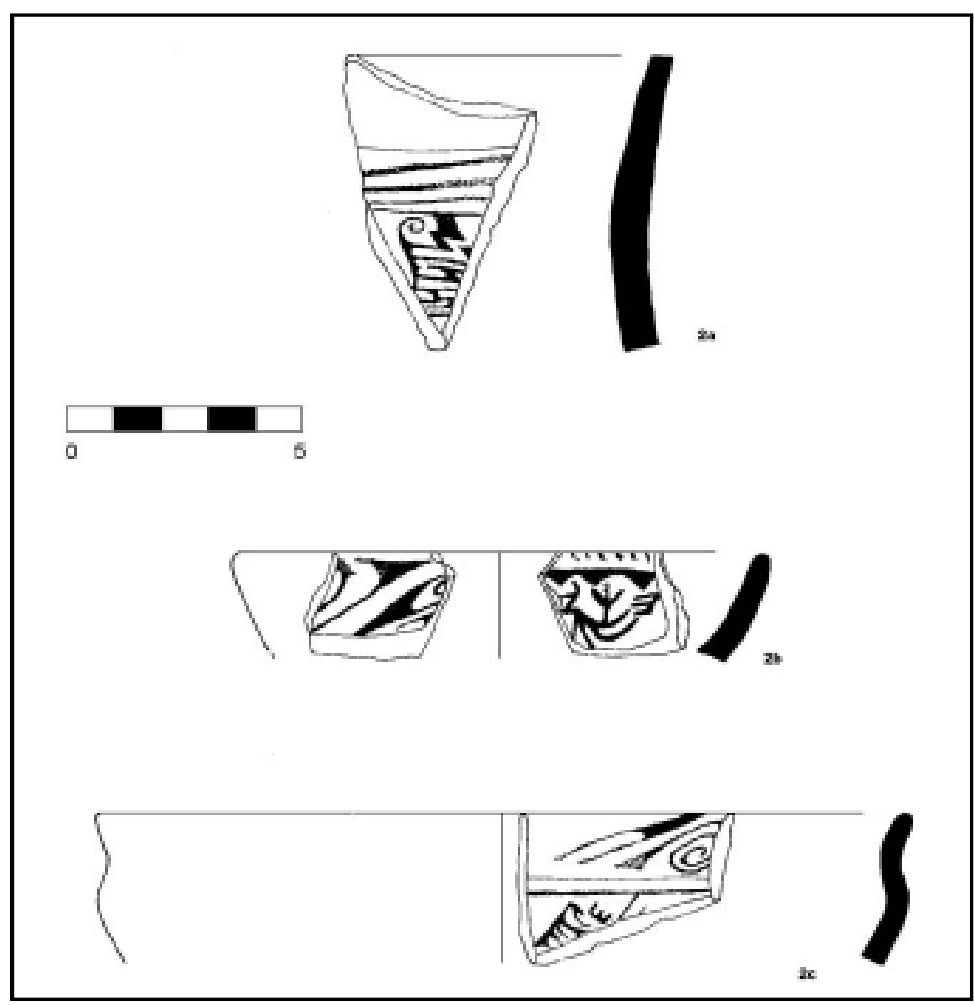

Figura 2A, 2B y 2C. Fragmentos Yavi-Chicha

\section{Cerámica Yavi-Chicha en tiempos incaicos.}

La cerámica Yavi-Chicha llega a su mayor influencia durante el período incaico, cuando la encontramos ampliamente distribuida no solo en su región nuclear sino entre el valle de Cinti en Bolivia (Rivera 2005), el Noroeste Argentino, incluyendo la zona de las Yungas argentinas (Cremonte et al. 2003, Garay de Fumagalli 2003, Nielsen 1996, Williams 2003) y las zonas chilenas del desierto de Atacama y el valle del río Loa (Uribe 2002).

La cerámica Yavi-Chicha del periodo inca (Fig. 2b, c) presenta diferentes variantes como el material Inca-Paya ${ }^{11}$, Yavi Chico Policromo, cerámica del tipo Inca Provincial y cerámica presentada como "pasta Chicha o Yavi" y diversas variantes de estilos Inca-Locales (Beierlein de Gutierrez 2007, Cremonte et al. 2003, Uribe 2002) y tanto Williams (2003) como D’Altroy et al. (1994) han señalado su importancia en tiempos incaicos. Aparte de haber sido usado en gran estilo como un bien de estatus en la región entre San Pedro de Atacama, el Altiplano de Sama, el Noroeste Argentino y posiblemente el valle de Tarija hay que señalar que no solo se copiaban características decorativas sino también rasgos tecnológicos para producir una cerámica similar a las vasijas Yavi-Chicha ${ }^{12}$. Este fenómeno se debe probablemente al uso como bien de estatus fomentado por los Incas, estableciendo el estilo Yavi-Chicha como una de las cerámicas de más alto prestigio en los Andes del Sur (Williams 2003). 
El porcentaje de la cerámica Yavi-Chicha en las regiones donde ésta es introducida en tiempos incaicos es muy bajo (Cremonte et al. 2003, 2005). Eso sostiene la hipótesis de que no se trate de grupos "originarios" del lugar sino de un bien de estatus producido y/o imitado localmente o introducido mediante las interacciones entre el estado inca, grupos de mitmaqkuna y las élites y grupos regionales/locales.

Las investigaciones en sitios adscritos etnohistóricamente a los Chichas han propuesto la posibilidad que algunos de ellos fueron poblaciones de mitmaqkuna Chichas mandados en resguardo de la frontera oriental inca. Revisaremos esta hipótesis en algunos ejemplos. Por un lado existe el caso de los fuertes de Huruncuta (Oroncota) y Cuzcotoro. Estos han podido ser ubicados en la cercanía del río Pilcomayo y la serranía Khosko Toro y Pärssinen y Siiriäinen (2003) mencionan material "Chicha" en ambos sitios. Este material cerámico, descrito brevemente como "una variante 'Chicha Blanco Monocromo (color blanco en ambos lados)" (ibd., pág. 174) difiere de lo que se conoce como Yavi-Chicha y es probable que Alconini (1998) tenga razón al sugerir que se trate de una tradición cerámica local todavía no descrita. En este caso tenemos sitios etnohistóricamente adscritos a los Chichas, pero con material cerámico ajeno a la cerámica Yavi-Chicha.

Por otra parte existe la fortaleza de Condorhuasi, identificada posiblemente en la serranía Santa Victoria (Beierlein 2004), donde los Chichas y Churumatas daban mit'a en calidad de guerreros según las fuentes escritas (Presta 1995). Aunque el sitio demuestra componentes incaicos en la cerámica y arquitectura, se distingue claramente la cerámica Yavi-Chicha existente en la zona. Eso hace suponer de una población de origen local o regional que habitaba la fortaleza. Ya que pertenece a la zona nuclear Chicha no puede ser interpretado como un lugar adonde se "mandaba" a los Chichas. Existe entonces un panorama complicado acerca de las diferentes fortalezas adscritas a los Chichas que solamente se podrá resolver con investigaciones específicas.

En tercer lugar, se ha interpretado la Cerámica Yavi-Chicha en contextos incaicos de los Yungas de la actual Argentina como presencia de grupos de mitmaqkuna Chichas, al mismo tiempo la hipótesis del uso del material Yavi Chico Policromo e Inca-Paya como bien de estatus ha adquirido mayor popularidad. Ambas interpretaciones explican el uso de esta cerámica no solo como indicador étnico sino también como bien de uso simbólico, identificador de relaciones de estatus entre un grupo local y el estado inca. Eso esta acorde a la idea de Lorandi (1984), quien propuso que grupos mitmaqkuna Chichas poseían un alto estatus jerárquico con posibles funciones de liderazgo entre diferentes grupos de mitmaqkuna y locales.

Estos tres ejemplos demuestran la dificultad de adscribir sitios arqueológicos a grupos étnicos mencionados en las fuentes. Aunque podemos identificar en algunos casos los sitios mencionados, el material arqueológico no es homogéneo, sino presenta más bien diferentes estilos cerámicos que no pueden ser adscritos simplemente a un "grupo étnico".

Sin embargo, el uso de las diferentes variantes de la cerámica Yavi-Chicha en estos contextos sociopolíticos de la frontera inca podría ser explicado usando el modelo de los grupos de identidad. En este caso las diferentes variedades de cerámica y sus contextos y porcentajes representarían un uso específico y situacional por parte de diferentes grupos, tomando en cuenta las poblaciones mitmaqkuna y entidades locales. 


\section{Discusión: ¿Los Chichas? Coincidencias y Rupturas.}

La revisión de los datos arqueológicos y etnohistóricos muestra a "Los Chichas", supuestamente homogéneos, como sujetos históricos y arqueológicos altamente variables y heterogéneos. Si revisamos la distribución del material cerámico Yavi-Chicha y los límites geográficos mencionados por las fuentes coloniales, veremos que se refieren un área nuclear común donde - arqueológicamente - la cerámica Yavi-Chicha prevalece desde un momento preincaico e - históricamente - los Chichas son denominados como "nación". Se trata de la región la Puna Argentina Septentrional, el valle del Río San Juan del Oro, el Altiplano de Sama y la parteSur del Altiplano de Lípez, lo que corresponde en parte con la región propuesta por Espinoza (1969) y Presta (1995). Sin embargo, este espacio nuclear no se presenta internamente homogéneo, sino demuestra la existencia de subgrupos identificables y no incluye el área de Calcha, una de las dos parcialidades Chichas según las fuentes.

Arqueológicamente, las variantes regionales y locales en la cerámica Yavi-Chicha en esta región han sido adscritas a una variabilidad interna, posiblemente basada en razones sociopolíticas (Angelo 1999). Según los datos publicados hasta el momento se puede observar una variabilidad inter e intraregional (Beierlein de Gutierrez 2007). Sin embargo, la presencia continua de diferentes variedades de cerámica Yavi-Chicha desde un momento preincaico es un indicador para un territorio usado por un grupo de identidad de larga duración, posiblemente asociable a la entidad cultural de las fuentes etnohistóricas denominado "Chichas". Ya que el desarrollo de esta cultura material parece remontarse al Horizonte Medio o los Desarrollos Regionales Tempranos (sensu Angelo 1999) podemos proponer esta región nuclear como el área central de lo que se denomina en el siglo XVI y XVII como "territorio Chicha".

Aparte de la zona nuclear, existen evidencias arqueológicas Yavi-Chicha en áreas que solo ofrecen datos esporádicos en las fuentes coloniales, pero donde los hallazgos arqueológicos indican una influencia Yavi-Chicha temprana, como Lípez, el desierto de Atacama y la cuenca del Río Loa. La cerámica Yavi-Chicha aparece alrededor del 1200-1300 d.C. en las zonas chilenas y desde el 900 d.C. en la Quebrada de Humahuaca, abriendo la posibilidad de interpretarlas como evidencias de una interacción sociopolítica que se podría haber iniciado en este momento (Fig. 1). Angelo (2006) propone que la variedad de hallazgos de la cerámica Chicha en diferentes contextos fuera de la zona nuclear y su variabilidad estilística dentro de la misma apuntan a una "activa reconfiguración" de las redes de interacción entre los Chichas y los habitantes de las áreas adyacentes en este momento, refiriéndose al modelo de la "movilidad giratoria".

Con la llegada de los Incas el panorama cambia, demostrando un aumento de cerámica Yavi-Chicha y sus variantes incaicas a nivel cuantitativo (Uribe 2002) y a nivel espacial. Así podemos observar una expansión en las regiones argentinas, posiblemente debido al uso de esta cerámica como bien de estatus. En este contexto es posible que la cerámica Yavi-Chicha haya sido usado en parte como indicador de etnicidad por grupos de mitmaqkuna Chichas, si seguimos las ideas de Barth de la intensificación de las expresiones étnicas en situaciones fronterizas. Por otra parte debe haber sido usada por grupos de identidad locales como indicador de estatus y representante de la ideología inca. Esta interpretación desde los datos arqueológicos está en concordancia con datos etnohistóricos acerca de la interacción Chicha con las regiones donde servían de mitmaqkuna. 


\section{Conclusión}

Usando datos arqueológicos y etnohistóricos, el panorama acerca de los Chichas y la cerámica Yavi-Chicha se vuelve mas detallado. Podemos observar que la presencia arqueológica y etnohistórica se sobreponen en varias regiones, enfocando la idea de una congruencia parcial entre la cerámica Yavi-Chicha y el grupo Chichas de las fuentes escritas. Sin embargo, una revisión de ambas fuentes presenta interesantes rupturas: Cuáles son las razones para la escasez de datos etnohistóricos acerca de la presencia Chicha en las regiones chilenas, donde su cultura material es parte integral del registro arqueológico desde fechas muy tempranas? Cuál es la explicación para la presencia etnohistórica de Chichas al Sur de Potosí, p.e. en Calcha, mientras que los datos arqueológicos nos hablan de una cultura material muy diferente a la Yavi-Chicha? Estas rupturas entre la etnohistoria y la arqueología conducen a preguntas acerca de la autorepresentación de los Chichas en las fuentes escritas y su autopercepción mediante su cultura material.

Propongo de manera tentativa una explicación de estas rupturas y coincidencias entre ambas fuentes mediante la presencia de diferentes grupos de identidad. En este caso es posible identificar los grupos de identidad mediante las diferentes variantes de la cerámica Yavi-Chicha, dejando de lado el tema de los diferentes intereses indígenas y europeos referentes a la autorepresentación de los Chichas en las fuentes.

Mientras que el área nuclear podría representar un grupo de identidad temporalmente estable expresado por cerámica Yavi-Chicha y posiblemente relacionado con parte del grupo Chichas de las fuentes tempranas, las regiones vecinas corresponden a identidades transeúntes e inestables. Es posible que la hipótesis de las redes de interacción se vea expresada en diferentes variantes de la cerámica Yavi-Chicha y su presencia en contextos muy diversos, siempre correspondientes con su uso situacional por grupos relacionados con el área nuclear y entidades locales en interacción con éstos. Ya que estas regiones no formaban parte del territorio nuclear no fueron representadas como "territorio Chichas" en las fuentes, aunque mantenían relaciones estrechas y de larga duración con el área nuclear.

La hipótesis de la cerámica Yavi-Chicha como un bien de estatus en el Noroeste Argentino durante el período inca, lleva a la posibilidad de que ésta hubiese circulado entre grupos de identidad ajenos al grupo del área nuclear, formando parte de una cultura material local sin relación alguna a los supuestos grupos "étnicos" Chichas. Se trataría de un bien cargado de significado simbólico, usado para expresar un estatus sociopolítico personal dentro de la administración inca, en vez de adscripciones a un grupo local o regional.

\section{Agradecimientos}

Quiero agradecer en especial a Raquel Gil Montero, quien me apoyó sin medida en el transcurso de la elaboración de este artículo. Mi gratitud a Roxana Boixadós, Daniel Gutiérrez, Karoline Noack y Gabriela Sica por sus valiosas críticas, revisiones y notas acerca de este texto. Y un agradecimiento especial a Daniel Gutierrez por la corrección del texto en español.

\section{Notas}

1. El término “Noroeste Argentino" se usa según la definición arqueológica de Bennett et al. (1948). 
2. Barragán (1994) propone puntos de interrelación de carácter lingüístico, sociopolítico y las denominaciones coloniales mismas. Aunque esto no responde la pregunta de las relaciones existentes, demuestra los paralelismos y congruencias entre estos grupos.

3. Saignes $(1985,1990)$ propone el valle del río Pilcomayo y de Tarija como posibles límites del territorio Chichas en tiempos incaicos.

4. "[...] en la provincia de Chichas, en Hurinsayo, el cacique Unichuca y el cacique Chapora y el cacique Condori y el cacique Talaua y el cacique Hallapa. En Anansuyo el cacique Chuchullamasa y el cacique Sindara y Yelma y Tucapa y el principal Caritima de Callao y el principal Arucapapa mitima de Ocolla y el principal Amanacache, mitima de Canche y el principal Condoricana, mitima de Pisquillata y el principal Malo mitima de Caranga y el principal Chicho mitima de Quillara y el principal Caguarca Pariaguana mitima de Condesuyo y el principal Chiara mitima de Collaga y el principal Chiara mitima de Aucachica mitima del Cuzco y el principal Tarraenrraua mitima de Tanbo y el principal Tasega mitima de Sura". (Varón Gabai 1997, pág. 245ff).

5. "y entro en la prouincia de los chichas y moyomoyos y amparais y aquitas copayapo churomatas y caracos y llego hasta los chiriguanos [y] hasta tucuman y alli hizo una fortaleza y puso muchos yndios mitimaes... y asi salieron a pocona y hicieron muchas fortalezas en el mesmo pocona y en sabaypata que es en los chiriguanas y en cuzcotuiro y pusso en todas las fortale[za]s muchos yndios de diuerssas partes [del tawantinsuyu] para guardasen la dha fortaleza y frontera a donde dexo muchos yndios orexones y al pressente estan poblados sus hijos y descendientes en las dhas fortale $[z a] s$ y fronteras-. y luego hallaron vna ffortaleza en la prouincia de los chuis y chichas llamada huruncuta y asolando aquella prouincia la poblo de muchos yndios orexones-."(Khipu del Capac Ayllu, en Pärssinen 2003, pág. 115).

6. Existe un debate intenso sobre el origen de los Churumatas, sus movimientos geográficos bajo los Incas y durante la colonia temprana. Su origen fue buscado en el Noroeste Argentino y el valle de Tarija, se les adscribió estatus de "señorío" (Espinoza 1985/86) y fueron presentados como grupo disperso, como "indios de arco y flecha" (Barragán 1994). Varios autores (Espinoza 1985/1986, Presta 1995, Saignes 1985) coinciden en que fueron usados como mitmaqkuna en el resguardo de las fortalezas orientales. Su relación con los Chichas fue atribuida a la relación establecida por los mandamientos incaicos de mit’a (Lorandi 1984, Schramm 1990)

7. Ver también Ávila (2005) para una síntesis.

8. Pareciera que el límite Este de la cerámica Yavi-Chicha en la parte boliviana fuese el Altiplano de Sama, ya que aquí se observa una interpretación propia del inventario decorativo Yavi-Chicha, basándose en las características tecnológicas descritas para este estilo (Beierlein 2004, 2006).

9. Nielsen (1996) relaciona la cerámica Yavi-Chicha con la fase Isla (aprox. 900-1250 d.C.) y Pukara (13501430 d.C.) en la Quebrada de Humahuaca, Uribe (2002) la describe para las fases Solor (1200-1470 d.C.) y Catarpe (1470-1535 d.C.) del Salar de Atacama y el Río Loa.

10. La cerámica de las tierras bajas ha sido trabajado intensivamente (Garay de Fumagalli 2003, Alconini y Rivera 2003). Sin embargo, no podemos ofrecer aún un panorama completo y estamos lejos de poder relacionarlo con grupos habitantes en esta zona. Esto se debe también en parte a la posibilidad de que se haya tratado de grupos altamente móviles que además fueron presionados por los constantes movimientos chiriguanos y de la reubicación bajo la influencia inca.

11. La cerámica Inca-Paya fue descrita por Bennett et al. (1948) como Casa Morada Polychrome, y fue redenominada La Paya por Serrano (1958). Existen diferentes variedades como Inca-La Paya (Williams 2003) y Yavi-La Paya (Uribe 1997).

12. D’Altroy et al. (1994) describen la imitación de la inclusión de lutitas blancas mediante el uso de vidrio volcánico, creando un aspecto similar al de la pasta Yavi-Chicha en Potrero-Chuquiago.

\section{Bibliografía}

Albeck, M. E.

2003. La Puna argentina en los períodos medio y tardío. Historia Argentina Prehispánica. Tomo 1 (ed. por E. Berberián y A. Nielsen), pp. 347-380. Editorial Brujas, Buenos Aires.

Alconini, S.

1998. Frontera Inka en el Chaco: Una aproximación a la Interacción Inka con los grupos GuaraníChiriguanos. Informe de trabajo, DINAAR, La Paz.

Alconini, S. y C. Rivera

2003. La tradición cerámica "estampada e incisa de bordes doblados" en la vertiente Oriental de los Andes: un caso de interacción e influencia desde las tierras bajas. La mitad verde del 
mundo andino:Investigaciones Arqueológicas en la Vertiente Oriental de los Andes y las Tierras Bajas de Bolivia y Argentina (ed. por G. Ortiz y B. Ventura), pp. 153-178. UnJU, Jujuy.

Angelo, D.

1999. Tráfico de Bienes, Minería y Aprovechamiento de Recursos en la región de los valles del Sur Boliviano (una aproximación arqueológica a la región de los Chichas, Prov. Sur Chichas-Potosí). Tesis de Licenciatura, UMSA, La Paz.

2006. Líneas de tiempo y círculos de consumo: acerca de las relaciones de interacción en el Altiplano Sur Andino. Esferas de interacción prehistóricas y fronteras nacionales modernas: los Andes sur centrales (ed. por H. Lechtman), pp. 127-166. IEP/IAR, Lima.

Angelo, D. y J. Capriles

2004. La importancia de las plantas psicotrópicas para la economíade intercambio y relaciones de interacción en el Altiplano Sur Andino. Chungará 36, supl. espect. 2: 10231035.

Arellano López, J.

1984. La Cultura Tarija: Aporte al conocimiento de las Señoríos Regionales del Sur Boliviano. Arqueología Boliviana 1: 73-78.

Ávila, F.

2005. El estilo alfarero Yavi y su relación con la construcción de entidades culturales. Theoria, vol. 14: 85-101.

Barragán, R.

1994. "IIndios de arco y flecha?”. Entre la historia y la Arqueología de las poblaciones del Norte de Chuquisaca. Ediciones ASUR 3, Sucre.

Barth, Fredrik (Ed.)

1969. Ethnic groups and boundaries. The social organization of cultural difference. Universitets Forlaget, Oslo.

Beierlein de Gutiérrez, M.

2000. Avances en la Arqueología de Tarija: El Material Arqueológico de la Reserva Biológica de la Cordillera de Sama. Ponencia, XIV RAE, La Paz.

2004. Archäologische Untersuchung der späten vorspanischen Besiedlung des Altiplano de Sama (Dept. Tarija, Bolivien): Die Fundorte Pucunayoj und El Fuerte. Tesis de Maestría no publicada, Universidad Libre de Berlín.

2006. La cerámica prehispánica tardía del Altiplano de Sama (Dept. Tarija, Bolivia) y su relación con las regiones adyacentes. Ponencia, $52^{\circ}$ Congreso Internacional de Americanistas, Sevilla.

2007. El Altiplano de Sama (Tarija, Bolivia) y su integración al ámbito Chicha-Inca: nuevos avances de investigación. Anales del XVI Congreso Nacional de Arqueología Argentina tomo 2, pp. 183-187. Jujuy

Bennett, W., E. F. Bleiler y F. H. Sommer

1948. Northwest Argentine Archaeology. Yale University Publications in Anthropology No. 38. 
Bernbeck, R.

1997. Theorien in der Archäologie. UTB für Wissenschaft, Francke Verlag, Tübingen und Basel.

Catacora, H., M. Clavijo, S. Fernández, P. Lima, F. Michel y M. Michel

2006. Una aproximación histórico-espacial a la relación hombre-medio ambiente en la cuenca del Poopó: el caso de quillacas. Diagnóstico de los recursos naturales y culturales de los lagos Poopo y Uru-Uru, Oruro, Bolivia (ed. por Rocha Olivio, O.), pp. 137-151. La Paz.

Cieza de León, $P$.

[1553]1984. Crónica del Perú. Primera Parte. Introducción de Franklin Pease G. Y. Fondo editorial de la Pontificia Universidad Católica del Perú, Lima.

Chilton, E. (Ed.)

1999. Material Meanings. Critical Approaches to the interpretation of material culture. University of Utah Press, Salt Lake City.

Condarco, C., E. Huarachi y M. Vargas

2003. Tras las huellas del Tambo Real de Paria. Fundación PIEB, La Paz.

Cook, N. D.

[1573]1975. Tasa de la Visita General de Francisco de Toledo. Introducción y versión paleográfica de Noble David Cook. Universidad Nacional Mayor de San Marcos, Lima.

Cornell, $\mathrm{P}$.

2004. Social identity, the body and power. Material Culture and other things. Post-disciplinary studies in the $21^{\text {st }}$ century (ed. por F. Fahlander \& T. Oestigaard), pp. 57-92. Gotarc, Series C, No. $\quad 61$, Göteborg.

Cremonte, M. B., M. Zaburlin y S. Peralta

2003. Agua Hedionda ¿un ejemplo de ocupación y control estatal? (Depto. San Antonio, Jujuy). Cuadernos 20: 109-132.

Cremonte, B., M. Garay de Fumagalli y G. Sica

2005 La frontera Oriental al sur de la Quebrada de Humahuaca. Un espacio conectivo. Mundo de Antes 4:51-66. Instituto de Arqueología y Museo. FCN e Inst. Miguel Lillo. UNT.

D’Altroy, T., A. M. Lorandi y V. Williams

1994. Producción y uso de cerámica en la economía política inka. Tecnología y organización de la producción de cerámica prehispánica en los Andes. (ed. por I. Shimada). Universidad

Católica del Perú, Lima.

Espinoza Soriano, W.

1969. El Memorial de Charcas. Crónica Inédita de 1582. Cantuta, Revista $\quad$ de $\quad l a$ Universidad de Educación.

1985/86. Los Churumatas y los mitmaes Chichas Orejones en los lindes del Collasuyo, siglos XV-XX. Revista Histórica 1985-1986, 35: 243-298. 
Fahlander, F. y T. Oestigaard

2004. Material culture and other things. Post-disciplinary studies in the $21^{\text {st }}$ century. Gotarc, Series C, No. 61, Göteborg.

Garay de Fumagalli, M.

2003. Del formativo al incaico, los valles Sudorientales de Jujuy en los procesos de interacción macroregionales. La mitad verde del mundo andino: Investigaciones Arqueológicas en la Vertiente Oriental de los Andes y las Tierras Bajas de Bolivia y Argentina (ed. por G. Ortiz y B. Ventura), pp. 229-260. UnJu, Jujuy.

Graham-Brown, P., S. Jones y C. Gamble (Ed.)

1996. Cultural Identity and Archaeology. The construction of European Communities. Routledge, London.

Hodder, I.

1982. Symbols in action. Ethnoarchaeological sudies of material culture. C a m b r i d g e University Press, Cambridge.

Ibarra Grasso, D. E. y R. Querejazu Lewis

1986. 30.000 años de Prehistoria en Bolivia. Los Amigos del Libro, Cochabamba.

Jones, $\mathrm{S}$.

1997. The archaeology of ethnicity. Constructing identities in past and present. Routledge, London.

Jones, S. y P. Graves-Brown

2006. Introduction: archaeology and cultural identity in Europe. Cultural Identity and Archaeology. The construction of European Communities ( ed. por Graham-Brown, P., S. Jones \& C. Gamble), pp.1-24. Routledge, London.

Julien, C.

1995. Oroncota entre dos mundos. Espacios, Etnias y Fronteras. Atenuaciones políticas en el Sur del Tawantinsuyu, Siglos XV-XVIII (ed. por A.M. Presta), pp. 97-160. ASUR 4, Sucre.

1999. History and Art in translation: the "paños" and other objects collected by Francisco de Toledo. Colonial Latin American Review, Vol. 8, 1: 61-89.

Julien, C., K. Angelis y Z. Bass Werner

1997. Historia de Tarija (Corpus Documental). Tomo VI. Editora Guadalquivir, Tarija.

Knapp, A.

1992. Archaeology, Annales and Ethnohistory. Cambridge University Press, Cambridge.

Krapovickas, $\mathrm{P}$.

1965. La cultura de Yavi, una nueva entidad cultural puneña. Etnia 2: 9-10.

1978. Los indios de la Puna en el siglo XVI. Relaciones XII: 71-93.

1984. Las poblaciones indígenas históricas del sector oriental de la Puna: un intento de correlación entre la información arqueológica y la etnográfica. Relaciones XV: 7-24. 
Krapovickas, P., C. P. Pla y S. Manuale

1989. Reconstruyendo el pasado: La Arqueología, la cultura de Yavi y los chichas. Revista de Antropología Año 4, No. 8: 3-11.

Lecoq, $P$.

1999. Uyuni Prehispanique. Archéologie de la Cordillére Intersalar (Sud-Ouest Bolivien). BAR International Series 798, Archaeopress, Oxford.

Michel, $\mathrm{M}$.

2000. El señorío prehispánico de Carangas. Ponencia presentada en el XV. Congreso de Arqueología Chilena.

Lorandi, A. M.

1984. Pleito de Juan Ochoa de Zarate por la posesión de los indios ocloyas. ¿Un caso de verticalidad étnica o un relicto de archipiélago estatal? Runa No. 14: 123-142.

Nielsen, A.

1996. Demografía y Cambio Social en Quebrada de Humahuaca (Jujuy, Argentina) 700-1535 d.C. Relaciones XXI: 307-354.

2002. Asentamientos, conflicto y cambio social en el altiplano de Lípez (Potosí). Revista Española de Antropología Americana 32: 179-205.

2003. Ocupaciones prehispánicas de la etapa agropastoril en la Laguna de V i 1 a m a (Jujuy, Argentina). Cuadernos 20: 81-108.

2004. Aproximación a la arqueología de la frontera tripartita Bolivia-Chile- Argentina. Chungará, Vol. 36, supl. espect. 2: 861-878.

Núñez, L. y T. Dillehay

[1979]1995. Movilidad giratoria, armonía social y desarrollo en los Andes Meridionales: Patrones de Tráfico e interacción económica. Universidad Católica del Norte, Antofagasta.

Pärssinen, $\mathrm{M}$.

2003. Tawantinsuyu: el estado Inca y su organización política. IFEA, Lima.

Pärssinen, M. y A. Siiriäinen

2003. Andes Orientales y Amazonía Occidental. Ensayos entre la historia y la arqueología de Bolivia, Brasil y Perú. Producciones Cima, La Paz.

Pease, F.

1995. Las Crónicas y los Andes. Pontificia Universidad Católica del Perú y Fondo de Cultura Económica, Lima.

Platt, T., T. Bouysse-Cassagne, O. Harris

2006. Qaraqara-Charka: Mallku, Inka y Rey en la provincia de Charcas (siglos XV-XVII); historia antropológica de una confederación aymara. IFEA, Lima. 
Presta, A. M.

1995. La población de los valles de Tarija, Siglo XVI. Aportes para la solución de un enigma etnohistórico en una frontera incaica. Espacios, Etnias y Fronteras. Atenuaciones políticas en el Sur del Tawantinsuyu, Siglos XV-XVIII (ed. por A.M. Presta), pp. 235-24. ASUR 4, Sucre.

Presta, A. M. (Ed.)

1995 Espacios, Etnias y Fronteras. Atenuaciones políticas en el Sur del Tawantinsuyu, Siglos XVXVIII. ASUR 4, Sucre.

Raffino, R., et al.

1993. Inka. Arqueología, Historia y Urbanismo del Altiplano Andino. Corregidor, Buenos Aires.

Renard-Casevitz, F.M. y T. Saignes

1988. Al este de los Andes. Relaciones entre las sociedades amazónicas y andinas entre los siglos XV y XVII. Abya-Yala/IFEA, Quito.

Rendón Lizarazu, P.

2004. Proyecto Arqueológico Tarija - Saire. Una aproximación a la arqueología de Tarija: el sitio Saire, estudio de las singularidades de su cerámica y la relación de esta con otros conjuntos. Tesis de Licenciatura, UMSA, La Paz.

Rivera Casanovas, C.

2005. Sociedades prehispánicas tardías en los valles interandinos del Suroeste de Chuquisaca, Bolivia. Nuevos Aportes No. 3: 76-92.

Saignes, $\mathrm{T}$.

1985. Los Andes orientales. Historia de un olvido. IFEA/Ceres, Cochabamba. 1990. Ava y Karai. Ensayos sobre la frontera chiriguano (siglos XVI-XX). Hisbol, La Paz.

Salomon, F. H.

1999. Testimonies: the making and reading of native south american historical sources. Cambridge History of the native people of the Americas, Vol. 3, part 1 (ed. por F. L. Salomon y S. Schwartz), pp. 19-95. Cambridge University Press, Cambridge.

Schramm, R.

[1560]1990. Visita de los yndios churumatas e yndios Charcas de Totora que todos estan en cabeza de su magestad. La Paz.

Serrano, A.

1958 Manual de cerámica indígena. Ediciones Assandri, Córdoba.

Shanks, M. y C. Tilley

1992. Re-Constructing Archaeology. Theory and Practice. Routledge, London.

Shennan, S.

1989. Introduction: Archaeological approaches to cultural identity. Archaeological approaches to cultural identity (ed. por S. Shennan), pp. 1-32. Routledge, London. 
Tarragó, $\mathrm{M}$.

1977. Relaciones prehispánicas entreSan Pedro de Atacama (Norte de Chile) y regiones aledañas: la quebrada de Humahuaca. Estudios Atacameños 5: 50-63.

1989. Contribución al Conocimiento Arqueológico de las Poblaciones de los Oasis de San Pedro de Atacama en relación con los Otros Pueblos Puneños, en especial el Sector Septentrional del Valle

Calchaquí. Tesis Doctoral citado en Uribe 2002. Universidad Nacional de Rosario, Rosario.

Tilley, C.

1989 Interpreting Material Culture. En: Hodder, Ian (Ed.) The Meanings of Things. Material Culture and Symbolic Expression. Unwin Hyman, London.

Uribe, M.

1997. La alfarería de Caspana en relación a la prehistoria tardía de la subárea circumpuneña. Estudios Atacameños 14: 243-262.

2002. Sobre alfarería, cementerios, fases y procesos durante prehistoria tardía del desierto de Atacama (800-1600 DC). Estudios Atacameños 22: 7-31.

Varón Gabai, R.

1997. Francisco Pizarro and his brothers. The illusion of power in sixteenth-century Peru. University of Oklahoma Press, Norman y London.

Vivar, G. de

1979[1558]. Crónica y relación copiosa y verdadera de los Reinos deChile. Colloquium Verlag, Berlín.

Williams, V.

2003. Poder y cultura material bajo el dominio inka. Ponencia, 1er Congreso de Historia, Santa Cruz de la Sierra.

2004. Nuevos datos sobre la prehistoria local en la Quebrada de Tolombón. Pcia. de Salta. Argentina. Local, Regional, Global (ed. por P. Cornell y P. Stenborg), pp. 163-209. GOTARC Series C, No. 54, Göteborg.

Wilson, $\mathrm{S}$.

1993. Structure and History: Combining Archaeology and Ethnohistory in the Contact Period Caribbean. Ethnohistory and Archaeology. Approaches to Postcontact Change in the Americas (ed. por D. Rogers y S. Wilson), pp. 19-30. Plenum Press, New York \& London.

Zanolli, C.

1995. Omaguaca: la tierra y su gente. Presencia chicha hacia el sur de Talina. Siglo XVI. Espacios, Etnias y Fronteras. Atenuaciones políticas en el Sur del Tawantinsuyu, Siglos XVXVIII (ed. por A. M. Presta), pp. 319-344. ASUR 4, Sucre.

2003. Los Chichas como mitimaes del Inca. Relaciones XXVIII: 45-60.

\section{Recursos de Internet:}

Cantarutti, G. y R. Mera

Sin año. Alfarería de la fase inca en el valle de Limarí: evidencias de influencia Yavi en jarros antropomórfos. En: galileo.ucn.cl/inv_arq_cuarta.htm. Acceso 23 de octubre 2006 
Williams, V.

Sin año. Horizonte Inca. En: http:/ / www.catamarcaguia.com.ar, Gobierno de la Provincia de Catamarca. Acceso 23 de octubre 2006. 